\title{
Existence of mild solution for a class of coupled systems of neutral fractional integro-differential equations with infinite delay in Banach space
}

\author{
Juqing Liu' and Kaihong Zhao ${ }^{2^{*}}$ (D)
}

\section{"Correspondence:}

zhaokaihongs@126.com

2Department of Applied

Mathematics, Kunming University of

Science and Technology, Kunming,

China

Full list of author information is

available at the end of the article

\begin{abstract}
We deal with a class of coupled systems of neutral fractional integro-differential equations with infinite delay in a Banach space in this paper. Based on the Banach contraction principle and Krasnoselskii's fixed point theorem, some new sufficient criteria are established to guarantee the existence of mild solutions. As an application, an example is given to illustrate the effectiveness of our main results.
\end{abstract}

MSC: 34K37; 34K40; 47H20

Keywords: Coupled system of neutral fractional integro-differential equations; Infinite delay; Existence of mild solution; Fixed point theorem

\section{Introduction}

Fractional differential equations have arisen as an excellent instrument for the description of hereditary properties of various processes and applications in plenty of fields such as porous medium, network, electro-magnetics, aerodynamics, visco-elasticity, ecology, bioscience, medicine biology, and so on. In the monograph [1], readers can find many mathematical models of fractional differential equations and their applications. Therefore, the study of fractional differential equation has attracted much attention and research. In recent years, many scholars have begun researching some new type of fractional differential equations, which include the neutral terms, integro-differential terms, or coupled terms. There have been some papers dealing with these fractional differential equations (see [235]). However, it is difficult and challenging to study this type of fractional differential equations since it involves the time delays and the abstract operators. So the purpose of this paper is to consider a class of coupled systems of neutral fractional integro-differential equations with infinite delay in a Banach space as follows:

$$
\left\{\begin{array}{l}
{ }^{c} D_{t}^{\alpha}\left[x(t)-g_{1}\left(t, x_{t}, y_{t}\right)\right]=\mathscr{A}\left[x(t)-g_{1}\left(t, x_{t}, y_{t}\right)\right]+f_{1}\left(t, x_{t}, y_{t}, \int_{0}^{t} e_{1}\left(t, s, x_{s}, y_{s}\right) d s\right), \\
{ }^{c} D_{t}^{\beta}\left[y(t)-g_{2}\left(t, x_{t}, y_{t}\right)\right]=\mathscr{B}\left[y(t)-g_{2}\left(t, x_{t}, y_{t}\right)\right]+f_{2}\left(t, x_{t}, y_{t}, \int_{0}^{t} e_{2}\left(t, s, x_{s}, y_{s}\right) d s\right), \\
x(t)=\phi_{1}(t) \in \mathcal{B}_{h}, y(t)=\phi_{2}(t) \in \mathcal{B}_{h}, \quad t \in(-\infty, 0],
\end{array}\right.
$$

(c) The Author(s) 2019. This article is distributed under the terms of the Creative Commons Attribution 4.0 International License (http://creativecommons.org/licenses/by/4.0/), which permits unrestricted use, distribution, and reproduction in any medium, provided you give appropriate credit to the original author(s) and the source, provide a link to the Creative Commons license, and indicate if changes were made. 
where $0<\alpha, \beta<1, J=[0, b],{ }^{c} D_{t}^{\alpha}$ and ${ }^{c} D_{t}^{\beta}$ denote the Caputo fractional derivatives. $\mathscr{A}$, $\mathscr{B}$ are the infinitesimal generators of a strongly continuous semigroup $\left\{\mathcal{T}_{\alpha}(t), t \geq 0\right\}$ and $\left\{\mathcal{T}_{\beta}(t), t \geq 0\right\}$ in a Banach space X, respectively. $g_{i}: J \times \mathcal{B}_{h}^{2} \rightarrow X, f_{i}: J \times \mathcal{B}_{h}^{2} \times X \rightarrow X, e_{i}:$ $J^{2} \times \mathcal{B}_{h}^{2} \rightarrow X(i=1,2)$ are three given continuous functions. We assume that the histories $x_{t}, y_{t}:(-\infty, 0] \rightarrow X, x_{t}(s)=x(t+s), y_{t}(s)=x(t+s), s \leq 0$, belong to an abstract phase space $\mathcal{B}_{h}$ characterized in Preliminaries. Two given initial functions $\phi_{1}, \phi_{2}$ also belong to an abstract phase space $\mathcal{B}_{h}$.

In addition, the main inspiration also comes from the literature [10, 13]. In [10], the authors investigated the existence of a mild solution for the following neutral fractional functional integro-differential equation with infinite delay:

$$
\left\{\begin{array}{l}
{ }^{c} D_{t}^{q}\left[x(t)-g\left(t, x_{t}\right)\right]=\mathscr{A} x(t)+f\left(t, x_{t}, \int_{0}^{t} h\left(t, s, x_{s}\right) d s\right), \quad t \in J=[0, b] \\
x(t)=\phi(t) \in \mathcal{B}_{h}, \quad t \in(-\infty, 0]
\end{array}\right.
$$

where ${ }^{c} D_{t}^{q}$ is the Caputo fractional derivative of order $0<q<1 . \mathscr{A}$ is the infinitesimal generator of a strongly continuous semigroup of $\{\mathcal{T}(t), t>0\}$ in a Banach space $X . f: J \times$ $\mathcal{B}_{h} \times X, g: J \times \mathcal{B}_{h}, h: J^{2} \times \mathcal{B}_{h}$ are given functions, where $\mathcal{B}_{h}$ is a phase space. The histories $x_{t}:(-\infty, 0] \rightarrow X$ defined by $x_{t}(s)=x(t+s), s \leq 0$, belong to an abstract space $\mathcal{B}_{h}$.

In [13], Zhang, Huang, and Liu researched the existence and uniqueness of a mild solution for the fractional equation with nonlocal conditions and infinite delay as follows:

$$
\left\{\begin{array}{l}
{ }^{c} D_{t}^{q}\left[x(t)-u\left(t, x_{t}\right)\right]=\mathscr{A}\left[x(t)-u\left(t, x_{t}\right)\right]+f\left(t, x_{t}, \int_{0}^{t} h\left(t, s, x_{s}\right) d s\right), \quad t \in J=[0, b], t \neq t_{k}, \\
\left.\Delta x\right|_{t=t_{k}}=I_{k}\left(x\left(t_{k}^{-}\right)\right), \quad k=1,2, \ldots, n, \\
x(t)=\phi(t) \in \mathcal{B}_{h}, \quad t \in(-\infty, 0]
\end{array}\right.
$$

where ${ }^{c} D_{t}^{q}$ is the Caputo fractional derivative of order $0<q<1$. The operator $\mathscr{A}$ generates a strongly continuous semigroup of bounded liner operators $\{\mathcal{T}(t), t>0\}$ in the Banach space $X$. And $u, f$ are two given continuous functions, $x(\cdot)$ belongs to the Banach space $X$, and $x_{t}(\cdot)$ denotes $x_{t}(\theta)=x(t+\theta), \theta \in(-\infty, 0] . I_{k}: X \rightarrow X,\left.\Delta x\right|_{t=t_{k}}=x\left(t_{k}^{+}\right)-x\left(t_{k}^{-}\right)$with $x\left(t_{k}^{+}\right)=\lim _{h \rightarrow 0^{+}} x\left(t_{k}+h\right), x\left(t_{k}^{-}\right)=\lim _{h \rightarrow 0^{-}} x\left(t_{k}+h\right), k=1,2, \ldots, n$, for $0=t_{0}<t_{1}<t_{2}<\cdots<$ $t_{n}<t_{n+1}=b$.

To the best our knowledge, there are rare papers dealing with the coupled system of neutral fractional integro-differential equations with infinite delay in a Banach space. Therefore, it is important and interesting to study the existence of a mild solution for system (1.1) by the fixed point theorem. Our results are based on the properties of the analytic semigroup and ideas and techniques.

The rest of this paper is organized as follows. In Sect. 2, we recall some definitions and lemmas including the Caputo fractional calculus, the Mittag-Leffler function, and the analytic semigroup. In Sect. 3, we shall prove the existence of solutions for system (1.1). In Sect. 4 , an example is given to demonstrate the application of our main results. Finally, the conclusion is given to simply recall our studies and results obtained in Sect. 5.

\section{Preliminaries}

In this section, we present some primary components, including notations, definitions, lemmas, and so on, which are required in the whole paper. 
First, we present the abstract phase space $\mathcal{B}_{h}$, which has been used in [36]. Assume that $h:(-\infty, 0] \rightarrow(0,+\infty)$ is a continuous function with $l=\int_{-\infty}^{0} h(t) d t<+\infty$. For any $c>0$, we define $\mathcal{B}=\{\psi:[-c, 0] \rightarrow X$ such that $\psi(t)$ is bounded and measurable $\}$ and equip with the norm $\|\psi\|_{[-c, 0]}=\sup _{s \in[-c, 0]}|\psi(s)|, \forall \psi \in \mathcal{B}$. Let us define

$$
\begin{aligned}
\mathcal{B}_{h}= & \left\{\psi:(-\infty, 0] \rightarrow X \text { such that for any } a>0,\left.\psi\right|_{[-a, 0]} \in \mathcal{B}\right. \\
& \text { and } \left.\int_{-\infty}^{0} h(s)\|\psi\|_{[s, 0]} d s<+\infty\right\} .
\end{aligned}
$$

If $\mathcal{B}_{h}$ is equipped with the norm $\|\psi\|_{\mathcal{B}_{h}}=\int_{-\infty}^{0} h(s)\|\psi\|_{[s, 0]} d s, \forall \psi \in \mathcal{B}_{h}$, then it is obvious that $\left(\mathcal{B}_{h},\|\cdot\|_{\mathcal{B}_{h}}\right)$ is a Banach space. Define

$$
\mathcal{B}_{h}^{\prime}=\left\{w=\left(w_{1}, w_{2}\right) \mid w_{i}:(-\infty, b] \rightarrow X \text { such that } w_{i}(0)=\phi_{i}(0) \in \mathcal{B}_{h}, i=1,2\right\}
$$

equipped with a seminorm $\|w\|_{\mathcal{B}_{h}^{\prime}}=\max \left\{\left\|\phi_{1}\right\|_{\mathcal{B}_{h}},\left\|\phi_{2}\right\|_{\mathcal{B}_{h}},\left\|w_{1}\right\|_{b},\left\|w_{2}\right\|_{b}\right\}$, where $\left\|w_{i}\right\|_{b}=$ $\sup _{s \in[0, b]}\left\{\left\|w_{i}(s)\right\|\right\}, i=1,2$.

Obviously, $\mathcal{B}_{h}^{\prime} \subset \mathbb{X}=X \times X$. For any $(x, y) \in \mathbb{X}$, define the seminorm $\|(x, y)\|=\|(x, y)\|_{\mathcal{B}_{h}^{\prime}}$. Thus $\mathcal{B}_{h}^{\prime}$ is a Banach space.

Lemma $2.1([10,36])$ Assume $w=\left(w_{1}, w_{2}\right) \in \mathcal{B}_{h}^{\prime}$, then for $t \in J, w_{i t} \in \mathcal{B}_{h}$. Moreover, $l\left|w_{i}(t)\right| \leq\left\|w_{i t}\right\|_{\mathcal{B}_{h}} \leq\left\|\phi_{i}\right\|_{\mathcal{B}_{h}}+l \sup _{s \in[0, t]}\left|w_{i}(s)\right|$, where $l=\int_{-\infty}^{0} h(t)<+\infty, i=1,2$.

Definition 2.1 ([37, 38]) The Riemann-Liouville fractional integral of order $\alpha$ of a continuous function $f:(a, \infty) \rightarrow R$ is given by

$$
I_{a^{+}}^{\alpha} f(t)=\frac{1}{\Gamma(\alpha)} \int_{a}^{t}(t-s)^{\alpha-1} f(s) d s,
$$

provided that the right-hand side is pointwise defined on $(a, \infty)$.

Definition $2.2([37,38])$ If $f \in C^{n}((a, \infty), R)$ and $\alpha>0$, then the Caputo fractional derivative of order $\alpha$ is given by

$$
{ }^{c} D_{a^{+}}^{\alpha} f(t)=\frac{1}{\Gamma(n-\alpha)} \int_{a}^{t} \frac{f^{(n)}(s)}{(t-s)^{\alpha-n+1}} d s,
$$

where $n-1<\alpha \leq n$, provided that the right-hand side is pointwise defined on $(a, \infty)$.

Lemma $2.2([37])$ Assume that $u \in C(a, b) \cap L(a, b)$ with a Caputo fractional derivative of order $\alpha>0$ that belongs to $C^{n}(a, b)$, then

$$
I_{a^{+}}^{\alpha c} D_{a^{+}}^{\alpha} u(t)=u(t)+c_{1}+c_{2}(t-a)+\cdots+c_{n}(t-a)^{n-1}
$$

for some $c_{i} \in R, i=0,1,2, \ldots, n-1$, where $n$ is the smallest integer greater than or equal to $\alpha$. 
Definition 2.3 ([37]) A two-parameter function of the Mittag-Leffler type is defined by the series expansion

$$
E_{\alpha, \beta}(z)=\sum_{k=0}^{\infty} \frac{z^{k}}{\Gamma(\alpha k+\beta)}=\frac{1}{2 \pi i} \int_{C} \frac{\lambda^{\alpha-\beta} e^{\lambda}}{\lambda^{\alpha}-z} d \lambda, \quad \alpha, \beta, z \in \mathbb{C}, \operatorname{Re}(\alpha)>0,
$$

where $C$ is a contour which starts and ends at $-\infty$ and encircles the disc $|\lambda| \leq \sqrt{|z|}$ counter clockwise. $\mathbb{C}$ is the complex field, $\operatorname{Re}(z)$ stands for the real part of complex number $z$.

For short, $E_{\alpha}(z)=E_{\alpha, 1}(z)$. It is an entire function which provides a simple generalization of the exponent function $E_{1}(z)=e^{z}$ and the cosine function $E_{2}\left(z^{2}\right)=\cosh (z), E_{2}\left(-z^{2}\right)=$ $\cos (z)$, and plays a vital role in the theory of fractional differential equations. The most interesting properties of the Mittag-Leffler functions are associated with their Laplace integral

$$
\int_{0}^{\infty} e^{-\lambda t} t^{\beta-1} E_{\alpha, \beta}\left(\omega t^{\alpha}\right) d t=\frac{\lambda^{\alpha-\beta}}{\lambda^{\alpha}-\omega}, \quad \operatorname{Re}(\lambda)>\omega^{\frac{1}{\alpha}}, \omega>0,
$$

and for more details, see [37].

Definition 2.4 ([39]) A closed and linear operator $\mathscr{A}$ is said to be sectorial if there are constants $\omega \in \mathbb{R}, \theta \in\left[\frac{\pi}{2}, \pi\right], M>0$ such that the following two conditions are satisfied:

(1) $\rho(\mathscr{A}) \subset S_{\omega, \theta}=\{\lambda \in \mathbb{C}: \lambda \neq \omega,|\arg (\lambda-\omega)|<\theta\}$,

(2) $\left\|(\lambda I-\mathscr{A})^{-1}\right\| \leq \frac{\mathcal{M}}{|\lambda-\omega|}, \lambda \in S_{\omega, \theta}$.

Definition 2.5 ([39]) Let $\mathscr{A}$ be a closed and linear operator with the domain $D(\mathscr{A})$ defined in a Banach space $X$. Let $\rho(\mathscr{A})$ be the resolvent set of $\mathscr{A} . \mathscr{A}$ is called the generator of an $\alpha$-resolvent family if there exist $\omega \geq 0$ and a strongly continuous function $\mathcal{T}_{\alpha}: \mathbb{R}_{+} \rightarrow \mathcal{L}(X)$, where $\mathcal{L}(X)$ is a Banach space of all bounded linear operators from $X$ into $X$ and the corresponding norm is denoted by $\|\cdot\|$ such that $\left\{\lambda^{\alpha}: \operatorname{Re}(\lambda)>\omega\right\} \subset \rho(\mathscr{A})$ and

$$
\left(\lambda^{\alpha} I-\mathscr{A}\right)^{-1} x=\int_{0}^{\infty} e^{\lambda t} \mathcal{T}_{\alpha}(t) x d t, \quad \operatorname{Re}(\lambda)>\omega, x \in X,
$$

where $\mathcal{T}_{\alpha}(t)$ is called the $\alpha$-resolvent family generated by $\mathscr{A}$.

Definition 2.6 ([39]) Let $\mathscr{A}$ be a closed and linear operator with the domain $D(\mathscr{A})$ defined in a Banach space $X$ and $\alpha>0$. We say that $\mathscr{A}$ is the generator of a solution operator if there exist $\omega \geq 0$ and a strongly continuous function $\mathcal{T}_{\alpha}: \mathbb{R}_{+} \rightarrow \mathcal{L}(X)$ such that $\left\{\lambda^{\alpha}: \operatorname{Re}(\lambda)>\omega\right\} \subset \rho(\mathscr{A})$ and

$$
\lambda^{\alpha-1}\left(\lambda^{\alpha} I-\mathscr{A}\right)^{-1} x=\int_{0}^{\infty} e^{\lambda t} \mathcal{T}_{\alpha}(t) x d t, \quad \operatorname{Re}(\lambda)>\omega, x \in X
$$

where $\mathcal{T}_{\alpha}(t)$ is called the solution operator generated by $\mathscr{A}$.

The concept of the solution operator is closely related to the concept of a resolvent family. For more details on $\alpha$-resolvent family and solution operators, we refer the reader to [38] and the references therein. 
Lemma 2.3 ([16]) If $E$ is a real Banach space and $F: E \rightarrow E$ is a contraction mapping, then $F$ has a unique fixed point in $E$.

Lemma 2.4 (Krasnoselskii's fixed point theorem [15]) Let B be a nonempty closed convex subset of a Banach space $(X,\|\cdot\|)$. Suppose that $P$ and $Q$ map B into $X$ such that

(i) $P x+Q x \in B$

(ii) $P$ is a contraction mapping;

(iii) $Q$ is continuous and compact.

Then there exists $z \in B$ such that $z=P z+Q z$.

Lemma 2.5 If the functions $g_{i}: J \times \mathcal{B}_{h}^{2} \rightarrow X, f_{i}: J \times \mathcal{B}_{h}^{2} \times X \rightarrow X, e_{i}: J^{2} \times \mathcal{B}_{h}^{2} \rightarrow X(i=$ $1,2)$ satisfy the uniform Hölder condition with exponent $\gamma \in(0,1], \mathscr{A}$ and $\mathscr{B}$ are sectorial operators, then a pair of solutions $(x, y) \in \mathbb{X}=X \times X$ of system (1.1) is given by the following coupled system of integral equations:

$$
\left\{\begin{aligned}
x(t)= & \mathcal{T}_{\alpha}(t)\left[\phi_{1}(0)-g_{1}\left(0, \phi_{1}(0), \phi_{2}(0)\right)\right]+g_{1}\left(t, x_{t}, y_{t}\right) \\
& +\int_{0}^{t} \mathcal{T}_{\alpha}(t-s) f_{1}\left(s, x_{s}, y_{s}, \int_{0}^{s} e_{1}\left(s, \tau, x_{\tau}, y_{\tau}\right) d \tau\right) d s, \quad t \in J, \\
y(t)= & \mathcal{T}_{\beta}(t)\left[\phi_{2}(0)-g_{2}\left(0, \phi_{1}(0), \phi_{2}(0)\right)\right]+g_{2}\left(t, x_{t}, y_{t}\right) \\
& +\int_{0}^{t} \mathcal{T}_{\beta}(t-s) f_{2}\left(s, x_{s}, y_{s} \int_{0}^{s} e_{2}\left(s, \tau, x_{\tau}, y_{\tau}\right) d \tau\right) d s, \quad t \in J
\end{aligned}\right.
$$

where

$$
\begin{aligned}
& \mathcal{T}_{\alpha}(t)=E_{\alpha, 1}\left(\mathscr{A} t^{\alpha}\right)=\frac{1}{2 \pi i} \int_{\hat{B}_{r}} e^{\lambda t} \frac{\lambda^{\alpha-1}}{\lambda^{\alpha}-\mathscr{A}} d \lambda, \\
& \mathcal{T}_{\beta}(t)=E_{\beta, 1}\left(\mathscr{B} t^{\alpha}\right)=\frac{1}{2 \pi i} \int_{\hat{B}_{r}} e^{\lambda t} \frac{\lambda^{\beta-1}}{\lambda^{\beta}-\mathscr{B}} d \lambda,
\end{aligned}
$$

here $\hat{B}_{r}$ denotes the Bromwich path; $\mathcal{T}_{\alpha}(t)$ and $\mathcal{T}_{\beta}(t)$ are the solution operators generated by $\mathscr{A}$ and $\mathscr{B}$, respectively.

Proof Assume that $(x, y) \in \mathbb{X}=X \times X$ is a pair of solutions of system (1.1), then we have for $t \in J$

$$
\left\{\begin{array}{l}
{ }^{c} D_{t}^{\alpha}\left[x(t)-g_{1}\left(t, x_{t}, y_{t}\right)\right]=\mathscr{A}\left[x(t)-g_{1}\left(t, x_{t}, y_{t}\right)\right]+f_{1}\left(t, x_{t}, y_{t} \int_{0}^{t} e_{1}\left(t, s, x_{s}, y_{s}\right) d s\right), \\
{ }^{c} D_{t}^{\beta}\left[y(t)-g_{2}\left(t, x_{t}, y_{t}\right)\right]=\mathscr{B}\left[y(t)-g_{2}\left(t, x_{t}, y_{t}\right)\right]+f_{2}\left(t, x_{t}, y_{t} \int_{0}^{t} e_{2}\left(t, s, x_{s}, y_{s}\right) d s\right) .
\end{array}\right.
$$

Applying Lemma 2.2, (2.2) gives

$$
\left\{\begin{aligned}
x(t)-g_{1}\left(t, x_{t}, y_{t}\right)= & c_{1}+\frac{1}{\Gamma(\alpha)} \int_{0}^{t}(t-s)^{\alpha-1} \mathscr{A}\left[x(s)-g_{1}\left(s, x_{s}, y_{s}\right)\right] d s \\
& +\frac{1}{\Gamma(\alpha)} \int_{0}^{t}(t-s)^{\alpha-1} f_{1}\left(s, x_{s}, y_{s}, \int_{0}^{s} e_{1}\left(s, \tau, x_{\tau}, y_{\tau}\right) d \tau\right) d s \\
y(t)-g_{2}\left(t, x_{t}, y_{t}\right)= & c_{2}+\frac{1}{\Gamma(\beta)} \int_{0}^{t}(t-s)^{\beta-1} \mathscr{B}\left[y(s)-g_{2}\left(s, x_{s}, y_{s}\right)\right] d s \\
& +\frac{1}{\Gamma(\beta)} \int_{0}^{t}(t-s)^{\beta-1} f_{2}\left(s, x_{s}, y_{s}, \int_{0}^{s} e_{2}\left(s, \tau, x_{\tau}, y_{\tau}\right) d \tau\right) d s .
\end{aligned}\right.
$$


By using the initial conditions $x(0)=\phi_{1}(0), y(0)=\phi_{2}(0)$, we get

$$
\left\{\begin{array}{l}
c_{1}=\phi_{1}(0)-g_{1}\left(0, \phi_{1}(0), \phi_{2}(0)\right), \\
c_{2}=\phi_{2}(0)-g_{2}\left(0, \phi_{1}(0), \phi_{2}(0)\right) .
\end{array}\right.
$$

Therefore,

$$
\left\{\begin{aligned}
x(t)- & g_{1}\left(t, x_{t}, y_{t}\right) \\
= & \phi_{1}(0)-g_{1}\left(0, \phi_{1}(0), \phi_{2}(0)\right)+\frac{1}{\Gamma(\alpha)} \int_{0}^{t}(t-s)^{\alpha-1} \mathscr{A}[x(s) \\
& \left.\quad-g_{1}\left(s, x_{s}, y_{s}\right)\right] d s+\frac{1}{\Gamma(\alpha)} \int_{0}^{t}(t-s)^{\alpha-1} f_{1}\left(s, x_{s}, y_{s}, \int_{0}^{s} e_{1}\left(s, \tau, x_{\tau}, y_{\tau}\right) d \tau\right) d s \\
y(t)- & g_{2}\left(t, x_{t}, y_{t}\right) \\
= & \phi_{2}(0)-g_{2}\left(0, \phi_{1}(0), \phi_{2}(0)\right)+\frac{1}{\Gamma(\beta)} \int_{0}^{t}(t-s)^{\beta-1} \mathscr{B}[y(s) \\
& \left.-g_{2}\left(s, x_{s}, y_{s}\right)\right] d s+\frac{1}{\Gamma(\beta)} \int_{0}^{t}(t-s)^{\beta-1} f_{2}\left(s, x_{s}, y_{s}, \int_{0}^{s} e_{2}\left(s, \tau, x_{\tau}, y_{\tau}\right) d \tau\right) d s .
\end{aligned}\right.
$$

Taking the Laplace transformation of system (2.4) on both sides, we obtain

$$
\left\{\begin{array}{l}
\mathscr{L}\left\{x(t)-g_{1}\left(t, x_{t}, y_{t}\right)\right\} \\
=\frac{1}{\lambda}\left[\phi_{1}(0)-g_{1}\left(0, \phi_{1}(0), \phi_{2}(0)\right)\right] \\
\quad+\frac{1}{\lambda^{\alpha}} \mathscr{A} \mathscr{L}\left\{x(t)-g_{1}\left(t, x_{t}, y_{t}\right)\right\}+\frac{1}{\lambda^{\alpha}} \mathscr{L}\left\{f_{1}\left(t, x_{t}, y_{t}, \int_{0}^{t} e_{1}\left(t, s, x_{s}, y_{s}\right) d s\right)\right\} \\
\mathscr{L}\left\{y(t)-g_{2}\left(t, x_{t}, y_{t}\right)\right\} \\
=\frac{1}{\lambda}\left[\phi_{2}(0)-g_{2}\left(0, \phi_{1}(0), \phi_{2}(0)\right)\right] \\
\quad+\frac{1}{\lambda^{\beta}} \mathscr{B} \mathscr{L}\left\{y(t)-g_{2}\left(t, x_{t}, y_{t}\right)\right\}+\frac{1}{\lambda^{\beta}} \mathscr{L}\left\{f_{2}\left(t, x_{t}, y_{t}, \int_{0}^{t} e_{2}\left(t, s, x_{s}, y_{s}\right) d s\right)\right\} .
\end{array}\right.
$$

Taking the inverse Laplace transformation on both sides of system (2.5), we have

$$
\left\{\begin{aligned}
x(t)= & \mathcal{T}_{\alpha}(t)\left[\phi_{1}(0)-g_{1}\left(0, \phi_{1}(0), \phi_{2}(0)\right)\right]+g_{1}\left(t, x_{t}, y_{t}\right) \\
& +\int_{0}^{t} \mathcal{T}_{\alpha}(t-s) f_{1}\left(s, x_{s}, y_{s}, \int_{0}^{s} e_{1}\left(s, \tau, x_{\tau}, y_{\tau}\right) d \tau\right) d s, \quad t \in J \\
y(t)= & \mathcal{T}_{\beta}(t)\left[\phi_{2}(0)-g_{2}\left(0, \phi_{1}(0), \phi_{2}(0)\right)\right]+g_{2}\left(t, x_{t}, y_{t}\right) \\
& +\int_{0}^{t} \mathcal{T}_{\beta}(t-s) f_{2}\left(s, x_{s}, y_{s} \int_{0}^{s} e_{2}\left(s, \tau, x_{\tau}, y_{\tau}\right) d \tau\right) d s, \quad t \in J
\end{aligned}\right.
$$

By the above computation, we obtain the mild solution defined by (2.1) satisfying (1.1). The proof is completed.

Definition 2.7 A pair of continuous functions $(x, y):(-\infty,+\infty) \rightarrow \mathbb{X}=X \times X$ is said to be a pair of mild solutions of system (1.1) if $x(0)=\phi_{1}(0) \in \mathcal{B}_{h}, y(0)=\phi_{2}(0) \in \mathcal{B}_{h}$, and $(x, y)$ satisfies the following coupled system (2.1) of integral equations.

\section{Existence of mild solutions}

In this section, we shall employ the fixed point theorems of the operator to prove the existence of a mild solution to system (1.1). According to Lemma 2.5, we define the operator $F: \mathbb{X} \rightarrow \mathbb{X}$ by

$$
F(x, y)(t)=\left(F_{1}(x, y)(t), F_{2}(x, y)(t)\right)^{T}, \quad \forall(x, y) \in \mathbb{X}, t \in(-\infty, b],
$$


where

$$
\begin{aligned}
& F_{1}(x, y)(t)=\left\{\begin{array}{cl}
\mathcal{T}_{\alpha}(t)\left[\phi_{1}(0)-g_{1}\left(0, \phi_{1}(0), \phi_{2}(0)\right)\right]+g_{1}\left(t, x_{t}, y_{t}\right) \\
\quad+\int_{0}^{t} \mathcal{T}_{\alpha}(t-s) f_{1}\left(s, x_{s}, y_{s}, \int_{0}^{s} e_{1}\left(s, \tau, x_{\tau}, y_{\tau}\right) d \tau\right) d s, & t \in J, \\
\phi_{1}(t), & t \in(-\infty, 0],
\end{array}\right. \\
& F_{2}(x, y)(t)=\left\{\begin{array}{cl}
\mathcal{T}_{\beta}(t)\left[\phi_{2}(0)-g_{2}\left(0, \phi_{1}(0), \phi_{2}(0)\right)\right]+g_{2}\left(t, x_{t}, y_{t}\right) \\
\quad+\int_{0}^{t} \mathcal{T}_{\beta}(t-s) f_{2}\left(s, x_{s}, y_{s} \int_{0}^{s} e_{2}\left(s, \tau, x_{\tau}, y_{\tau}\right) d \tau\right) d s, & t \in J, \\
\phi_{2}, & t \in(-\infty, 0] .
\end{array}\right.
\end{aligned}
$$

So the existence of solutions for system (1.1) is equivalent to the existence of the fixed point for the operator $F$ defined by (3.1)-(3.3). Next we shall prove our main results.

Theorem 3.1 Assume that conditions $\left(H_{1}\right)-\left(H_{5}\right)$ hold. Then system (1.1) has a pair of unique mild solutions $\left(x^{*}, y^{*}\right) \in \mathbb{X}$.

$\left(H_{1}\right)$ The strongly continuous semigroups of bounded linear operators $\mathcal{T}_{\alpha}(t)$ and $\mathcal{T}_{\beta}(t)$ generated by $\mathscr{A}$ and $\mathscr{B}$ are all compact, and there exist two positive constants $N_{1}$ and $N_{2}$ such that the modules of $\mathcal{T}_{\alpha}(t)$ and $\mathcal{T}_{\beta}(t)$ satisfy

$$
\left\|\mathcal{T}_{\alpha}(t)\right\| \leq N_{1}, \quad\left\|\mathcal{T}_{\beta}(t)\right\| \leq N_{2}, \quad \forall t \in J
$$

$\left(H_{2}\right)$ The functions $g_{i}: J \times \mathcal{B}_{h}^{2} \rightarrow X(i=1,2)$ are the continuous functions, and there exist some positive constants $a_{i 1}$ and $a_{i 2}$ such that, for all $t \in J, x_{t}, y_{t}, \bar{x}_{t}, \bar{y}_{t} \in \mathcal{B}_{h}$

$$
\left\|g_{i}\left(t, x_{t}, y_{t}\right)-g_{i}\left(t, \bar{x}_{t}, \bar{y}_{t}\right)\right\| \leq a_{i 1}\left\|x_{t}-\bar{x}_{t}\right\|_{\mathcal{B}_{h}}+a_{i 2}\left\|y_{t}-\bar{y}_{t}\right\|_{\mathcal{B}_{h}} .
$$

$\left(H_{3}\right)$ The functions $f_{i}: J \times \mathcal{B}_{h}^{2} \times X \rightarrow X(i=1,2)$ are the continuous functions, and there exist some positive constants $b_{i 1}, b_{i 2}$, and $b_{i 3}$ such that for all $t \in J, x_{t}, y_{t}, \bar{x}_{t}, \bar{y}_{t} \in \mathcal{B}_{h}$, $u, v \in X$

$$
\left\|f_{i}\left(t, x_{t}, y_{t}, u\right)-f_{i}\left(t, \bar{x}_{t}, \bar{y}_{t}, v\right)\right\| \leq b_{i 1}\left\|x_{t}-\bar{x}_{t}\right\|_{\mathcal{B}_{h}}+b_{i 2}\left\|y_{t}-\bar{y}_{t}\right\|_{\mathcal{B}_{h}}+b_{i 3}\|u-v\| .
$$

$\left(H_{4}\right)$ The functions $e_{i}: J^{2} \times \mathcal{B}_{h}^{2} \rightarrow X(i=1,2)$ are the continuous functions, and there exist some positive constants $c_{i 1}$ and $c_{i 2}$ such that for all $t, s \in J, x_{s}, y_{s}, \bar{x}_{s}, \bar{y}_{s} \in \mathcal{B}_{h}$

$$
\left\|e_{i}\left(t, s, x_{s}, y_{s}\right)-e_{i}\left(t, s, \bar{x}_{s}, \bar{y}_{s}\right)\right\| \leq c_{i 1}\left\|x_{s}-\bar{x}_{s}\right\|_{\mathcal{B}_{h}}+c_{i 2}\left\|y_{s}-\bar{y}_{s}\right\|_{\mathcal{B}_{h}} .
$$

(H) $l\left[a_{i 1}+a_{i 2}+b N_{i}\left(b_{i 1}+b_{12}\right)+b^{2} N_{i} b_{i 3}\left(c_{i 1}+c_{i 2}\right)\right]<1, i=1,2$, where $l=\int_{-\infty}^{0} h(s) d s$.

Proof Now we use Lemma 2.3 to prove that $F: \mathbb{X} \rightarrow \mathbb{X}$ defined by (3.1)-(3.3) has a unique fixed point. In fact, for all $t \in(-\infty, b],(x, y),(\bar{x}, \bar{y}) \in \mathbb{X}$, when $t \in(-\infty, 0]$, we have

$$
F(x, y)(t)-F(\bar{x}, \bar{y})(t)=\left(\phi_{1}(t)-\phi_{1}(t), \phi_{2}(t)-\phi_{2}(t)\right)=(0,0),
$$

which implies that

$$
\|F(x, y)(t)-F(\bar{x}, \bar{y})(t)\|=0
$$


When $t \in J=[0, b]$, it follows from $\left(H_{1}\right)-\left(H_{4}\right)$ that

$$
\begin{aligned}
& \left\|F_{1}(x, y)(t)-F_{1}(\bar{x}, \bar{y})(t)\right\| \\
& =\| g_{1}\left(t, x_{t}, y_{t}\right)-g_{1}\left(t, \bar{x}_{t}, \bar{y}_{t}\right)+\int_{0}^{t} \mathcal{T}_{\alpha}(t-s) \\
& \times\left[f_{1}\left(s, x_{s}, y_{s}, \int_{0}^{s} e_{1}\left(s, \tau, x_{\tau}, y_{\tau}\right) d \tau\right) d s-f_{1}\left(s, \bar{x}_{s}, \bar{y}_{s}, \int_{0}^{s} e_{1}\left(s, \tau, \bar{x}_{\tau}, \bar{y}_{\tau}\right) d \tau\right) d s\right] \| \\
& \leq\left\|g_{1}\left(t, x_{t}, y_{t}\right)-g_{1}\left(t, \bar{x}_{t}, \bar{y}_{t}\right)\right\|+\int_{0}^{b}\left|\mathcal{T}_{\alpha}(t-s)\right| \| f_{1}\left(s, x_{s}, y_{s}, \int_{0}^{s} e_{1}\left(s, \tau, x_{\tau}, y_{\tau}\right) d \tau\right) d s \\
& -f_{1}\left(s, \bar{x}_{s}, \bar{y}_{s}, \int_{0}^{s} e_{1}\left(s, \tau, \bar{x}_{\tau}, \bar{y}_{\tau}\right) d \tau\right) d s \| \\
& \leq\left\|g_{1}\left(t, x_{t}, y_{t}\right)-g_{1}\left(t, \bar{x}_{t}, \bar{y}_{t}\right)\right\|+\int_{0}^{b}\left|\mathcal{T}_{\alpha}(t-s)\right| \| f_{1}\left(s, x_{s}, y_{s}, \int_{0}^{s} e_{1}\left(s, \tau, x_{\tau}, y_{\tau}\right) d \tau\right) d s \\
& -f_{1}\left(s, \bar{x}_{s}, \bar{y}_{s}, \int_{0}^{s} e_{1}\left(s, \tau, \bar{x}_{\tau}, \bar{y}_{\tau}\right) d \tau\right) d s \| \\
& \leq a_{11}\left\|x_{t}-\bar{x}_{t}\right\|_{\mathcal{B}_{h}}+a_{12}\left\|y_{t}-\bar{y}_{t}\right\|_{\mathcal{B}_{h}}+N_{1} \int_{0}^{b}\left[b_{11}\left\|x_{s}-\bar{x}_{s}\right\|_{\mathcal{B}_{h}}+b_{12}\left\|y_{s}-\bar{y}_{s}\right\|_{\mathcal{B}_{h}}\right. \\
& \left.+b_{13}\left\|\int_{0}^{s}\left[e_{1}\left(s, \tau, x_{\tau}, y_{\tau}\right)-e_{1}\left(s, \tau, \bar{x}_{\tau}, \bar{y}_{\tau}\right)\right] d \tau\right\|\right] d s \\
& \leq a_{11}\left\|x_{t}-\bar{x}_{t}\right\|_{\mathcal{B}_{h}}+a_{12}\left\|y_{t}-\bar{y}_{t}\right\|_{\mathcal{B}_{h}}+N_{1} \int_{0}^{b}\left[b_{11}\left\|x_{s}-\bar{x}_{s}\right\|_{\mathcal{B}_{h}}+b_{12}\left\|y_{s}-\bar{y}_{s}\right\|_{\mathcal{B}_{h}}\right. \\
& \left.+b_{13} \int_{0}^{s}\left[c_{11}\left\|x_{\tau}-\bar{x}_{\tau}\right\|_{\mathcal{B}_{h}}+c_{12}\left\|y_{\tau}-\bar{y}_{\tau}\right\|_{\mathcal{B}_{h}}\right] d \tau\right] d s \\
& \leq l a_{11}\|x-\bar{x}\|_{b}+l a_{12}\|y-\bar{y}\|_{b}+N_{1} \int_{0}^{b}\left[l b_{11}\|x-\bar{x}\|_{b}+l b_{12}\|y-\bar{y}\|_{b}\right. \\
& \left.+b_{13} \int_{0}^{b}\left[l c_{11}\|x-\bar{x}\|_{b}+l c_{12}\|y-\bar{y}\|_{b}\right] d \tau\right] d s \\
& =l a_{11}\|x-\bar{x}\|_{b}+l a_{12}\|y-\bar{y}\|_{b}+b N_{1}\left[l b_{11}\|x-\bar{x}\|_{b}+l b_{12}\|y-\bar{y}\|_{b}\right. \\
& \left.+b b_{13}\left[l c_{11}\|x-\bar{x}\|_{b}+l c_{12}\|y-\bar{y}\|_{b}\right]\right] \\
& \leq l\left[a_{11}+a_{12}+b N_{1}\left(b_{11}+b_{12}\right)+b^{2} N_{1} b_{13}\left(c_{11}+c_{12}\right)\right]\|(x-\bar{x}, y-\bar{y})\| \\
& <\|(x-\bar{x}, y-\bar{y})\| \text {. }
\end{aligned}
$$

Similarly, we have

$$
\begin{aligned}
& \left\|F_{2}(x, y)(t)-F_{2}(\bar{x}, \bar{y})(t)\right\| \\
& \quad \leq l\left[a_{21}+a_{22}+b N_{2}\left(b_{21}+b_{22}\right)+b^{2} N_{2} b_{23}\left(c_{21}+c_{22}\right)\right]\|(x-\bar{x}, y-\bar{y})\| \\
& \quad<\|(x-\bar{x}, y-\bar{y})\|, \quad t \in J=[0, b] .
\end{aligned}
$$

In view of $\left(H_{5}\right)$ and (3.4)-(3.6), we know that $F$ defined by (3.1)-(3.3) is a contraction mapping. Then $F$ has a unique fixed point $\left(x^{*}, y^{*}\right) \in \mathbb{X}$. Therefore, system (1.1) has a pair of unique mild solutions $\left(x^{*}, y^{*}\right) \in \mathbb{X}$. The proof of Theorem 3.1 is completed. 
Theorem 3.2 Assume that $\left(H_{1}\right)-\left(H_{2}\right)$ hold. If the following conditions $\left(H_{6}\right)-\left(H_{8}\right)$ hold, then system (1.1) has at least a pair of mild solutions.

$\left(H_{6}\right)$ The functions $f_{i}: J \times \mathcal{B}_{h}^{2} \times X \rightarrow X(i=1,2)$ are the continuous functions, and there exist some positive constants $B_{i 1}, B_{i 2}$, and $B_{i 3}$ such that for all $t \in J, x_{t}, y_{t} \in \mathcal{B}_{h}, u \in X$

$$
\left\|f_{i}\left(t, x_{t}, y_{t}, u\right)\right\| \leq B_{i 1}\left\|x_{t}\right\|_{\mathcal{B}_{h}}+B_{i 2}\left\|y_{t}\right\|_{\mathcal{B}_{h}}+B_{i 3}\|u\| .
$$

$\left(H_{7}\right)$ The functions $e_{i}: J^{2} \times \mathcal{B}_{h}^{2} \rightarrow X(i=1,2)$ are the continuous functions, and there exist some positive constants $C_{i 1}$ and $C_{i 2}$ such that for all $t, s \in J, x_{s}, y_{s} \in \mathcal{B}_{h}$

$$
\left\|e_{i}\left(t, s, x_{s}, y_{s}\right)\right\| \leq C_{i 1}\left\|x_{s}\right\|_{\mathcal{B}_{h}}+C_{i 2}\left\|y_{s}\right\|_{\mathcal{B}_{h}}
$$

$\left(H_{8}\right) \varrho_{i}=N_{i}+\left(l+N_{i}\right)\left(a_{i 1}+a_{i 2}\right)+l b N_{i}\left(B_{i 1}+B_{i 2}\right)+l b^{2} N_{i} B_{i 3}\left(C_{i 1}+C_{i 2}\right)<1, i=1,2$, where $l=\int_{-\infty}^{0} h(s) d s$.

Proof Let $r=\max \left\{\frac{\left(1+N_{1}\right) \sup _{t \in J}\left\|g_{1}(t, 0,0)\right\|}{1-\varrho_{1}}, \frac{\left(1+N_{2}\right) \sup _{t \in J}\left\|g_{2}(t, 0,0)\right\|}{1-\varrho_{2}}\right\}, \bar{\Omega}=\{(x, y) \in \mathbb{X}:\|(x, y)\| \leq r\}$, where $\|(x, y)\|=\|(x, y)\|_{\mathcal{B}_{h}^{\prime}}$ Clearly, $\bar{\Omega}$ is a nonempty closed convex subset of a Banach space $\mathbb{X}$. Define the operator $F: \mathbb{X} \rightarrow \mathbb{X}$ as (3.1)-(3.3). We split $F=P+Q$ such that, for all $t \in(-\infty, b],(x, y) \in \mathbb{X}$,

$$
\begin{aligned}
& P_{1}(x, y)(t)= \begin{cases}\phi_{1}(t), & t \in(-\infty, 0], \\
\mathcal{T}_{\alpha}(t)\left[\phi_{1}(0)-g_{1}\left(0, \phi_{1}(0), \phi_{2}(0)\right)\right]+g_{1}\left(t, x_{t}, y_{t}\right), & t \in J,\end{cases} \\
& P_{2}(x, y)(t)= \begin{cases}\phi_{2}(t), & t \in(-\infty, 0], \\
\mathcal{T}_{\beta}(t)\left[\phi_{2}(0)-g_{2}\left(0, \phi_{1}(0), \phi_{2}(0)\right)\right]+g_{2}\left(t, x_{t}, y_{t}\right), & t \in J,\end{cases} \\
& Q_{1}(x, y)(t)= \begin{cases}0, & t \in(-\infty, 0], \\
\int_{0}^{t} \mathcal{T}_{\alpha}(t-s) f_{1}\left(s, x_{s}, y_{s}, \int_{0}^{s} e_{1}\left(s, \tau, x_{\tau}, y_{\tau}\right) d \tau\right) d s, & t \in J,\end{cases} \\
& Q_{2}(x, y)(t)= \begin{cases}0, & t \in(-\infty, 0], \\
\int_{0}^{t} \mathcal{T}_{\beta}(t-s) f_{2}\left(s, x_{s}, y_{s} \int_{0}^{s} e_{2}\left(s, \tau, x_{\tau}, y_{\tau}\right) d \tau\right) d s, & t \in J .\end{cases}
\end{aligned}
$$

When $t \in(-\infty, 0]$, for all $(x, y) \in \bar{\Omega}$, we have

$$
\|F(x, y)(t)\|=\left\|\left(\phi_{1}, \phi_{2}\right)\right\|=\max \left\{\left\|\phi_{1}\right\|_{\mathcal{B}_{h}},\left\|\phi_{2}\right\|_{\mathcal{B}_{h}}\right\} \leq\|(x, y)\| \leq r .
$$

When $t \in J=[0, b]$, for all $(x, y) \in \bar{\Omega}$, it follows from $\left(H_{1}\right)-\left(H_{2}\right)$ and $\left(H_{6}\right)-\left(H_{8}\right)$ that

$$
\begin{aligned}
& \left\|F_{1}(x, y)(t)\right\| \\
& =\| \mathcal{T}_{\alpha}(t)\left[\phi_{1}(0)-g_{1}\left(0, \phi_{1}(0), \phi_{2}(0)\right)\right]+g_{1}\left(t, x_{t}, y_{t}\right) \\
& \quad+\int_{0}^{t} \mathcal{T}_{\alpha}(t-s) f_{1}\left(s, x_{s}, y_{s}, \int_{0}^{s} e_{1}\left(s, \tau, x_{\tau}, y_{\tau}\right) d \tau\right) d s \| \\
& =\| \mathcal{T}_{\alpha}(t)\left[\phi_{1}(0)+g_{1}(0,0,0)-\left(g_{1}\left(0, \phi_{1}(0), \phi_{2}(0)\right)-g_{1}(0,0,0)\right)\right]
\end{aligned}
$$




$$
\begin{aligned}
& +\left(g_{1}\left(t, x_{t}, y_{t}\right)-g_{1}(t, 0,0)\right)+g_{1}(t, 0,0) \\
& +\int_{0}^{t} \mathcal{T}_{\alpha}(t-s) f_{1}\left(s, x_{s}, y_{s}, \int_{0}^{s} e_{1}\left(s, \tau, x_{\tau}, y_{\tau}\right) d \tau\right) d s \| \\
\leq & \left|\mathcal{T}_{\alpha}(t)\right|\left[\left\|\phi_{1}\right\|_{\mathcal{B}_{h}}+\left\|g_{1}(0,0,0)\right\|+a_{11}\left\|\phi_{1}\right\|_{\mathcal{B}_{h}}+a_{12}\left\|\phi_{2}\right\|_{\mathcal{B}_{h}}\right] \\
& +\sup _{t \in J}\left\|g_{1}(t, 0,0)\right\|+a_{11}\left\|x_{t}\right\|_{\mathcal{B}_{h}}+a_{12}\left\|y_{t}\right\|_{\mathcal{B}_{h}} \\
& +\int_{0}^{b} \mid \mathcal{T}_{\alpha}(t-s) \|\left[B_{11}\left\|x_{s}\right\|_{\mathcal{B}_{h}}+B_{12}\left\|y_{s}\right\|_{\mathcal{B}_{h}}+B_{13} \int_{0}^{b}\left\|e_{1}\left(s, \tau, x_{\tau}, y_{\tau}\right)\right\| d \tau\right] d s \\
\leq & N_{1}\left[\left\|\phi_{1}\right\|_{\mathcal{B}_{h}}+\sup _{t \in J}\left\|g_{1}(t, 0,0)\right\|+a_{11}\left\|\phi_{1}\right\|_{\mathcal{B}_{h}}+a_{12}\left\|\phi_{2}\right\|_{\mathcal{B}_{h}}\right] \\
& +\sup _{t \in J}\left\|g_{1}(t, 0,0)\right\|+a_{11}\left\|x_{t}\right\|_{\mathcal{B}_{h}}+a_{12}\left\|y_{t}\right\|_{\mathcal{B}_{h}} \\
& +b N_{1}\left[B_{11}\left\|x_{s}\right\|_{\mathcal{B}_{h}}+B_{12}\left\|y_{s}\right\|_{\mathcal{B}_{h}}+b B_{13}\left(C_{11}\left\|x_{\tau}\right\|_{\mathcal{B}_{h}}+C_{12}\left\|y_{\tau}\right\|_{\mathcal{B}_{h}}\right)\right] \\
\leq & N_{1}\left[\left\|\phi_{1}\right\|_{\mathcal{B}_{h}}+\sup _{t \in J}\left\|g_{1}(t, 0,0)\right\|+a_{11}\left\|\phi_{1}\right\|_{\mathcal{B}_{h}}+a_{12}\left\|\phi_{2}\right\|_{\mathcal{B}_{h}}\right] \\
& +\sup _{t \in J}\left\|g_{1}(t, 0,0)\right\|+l a_{11}\|x\|_{b}+l a_{12}\|y\|_{b}+b N_{1}\left[l B_{11}\|x\|_{b}+l B_{12}\|y\|_{b}\right. \\
& \left.+b B_{13}\left(l C_{11}\|x\|_{b}+l C_{12}\|y\|_{b}\right)\right] \\
\leq & {\left[N_{1}+\left(l+N_{1}\right)\left(a_{11}+a_{12}\right)+l b N_{1}\left(B_{11}+B_{12}\right)+l b^{2} N_{1} B_{13}\left(C_{11}+C_{12}\right)\right]\|(x, y)\| } \\
& +\left(1+N_{1}\right) \sup _{t \in J}\left\|g_{1}(t, 0,0)\right\|=\varrho_{1}\|(x, y)\|+\left(1+N_{1}\right) \sup _{t \in J}\left\|g_{1}(t, 0,0)\right\| \\
\leq & r .
\end{aligned}
$$

Similarly, we also get

$$
\begin{aligned}
& \left\|F_{2}(x, y)(t)\right\| \\
& \leq \quad\left[N_{2}+\left(l+N_{2}\right)\left(a_{21}+a_{22}\right)+l b N_{2}\left(B_{21}+B_{22}\right)+l b^{2} N_{2} B_{23}\left(C_{21}+C_{22}\right)\right]\|(x, y)\| \\
& \quad+\left(1+N_{2}\right) \sup _{t \in J}\left\|g_{2}(t, 0,0)\right\|=\varrho_{2}\|(x, y)\|+\left(1+M_{2}\right) \sup _{t \in J}\left\|g_{2}(t, 0,0)\right\| \\
& \leq r .
\end{aligned}
$$

(3.11)-(3.13) indicate that $P(x, y)+Q(x, y) \in \bar{\Omega}$, that is, condition (i) of Lemma 2.4 holds. Similar to (3.11)-(3.13), we also prove that $P$ and $Q$ map $\bar{\Omega}$ into $\bar{\Omega} \subset X$, and $Q$ is uniformly bounded. Next we only need to show that $P$ is a contraction mapping, and $Q$ is equicontinuous. Indeed, for all $(x, y),(\bar{x}, \bar{y}) \in \bar{\Omega}$, when $t \in(-\infty, 0], P(x, y)(t)-P(\bar{x}, \bar{y})(t)=$ $\left(\phi_{1}(t)-\phi_{1}(t), \phi_{2}(t)-\phi_{2}(t)\right)=(0,0)$, which leads to

$$
\|P(x, y)(t)-P(\bar{x}, \bar{y})(t)\|=0 .
$$

When $t \in J=[0, b]$, from (3.7)-(3.8), $\left(H_{2}\right)$, and $\left(H_{8}\right)$, we have

$$
\begin{aligned}
& \left\|P_{i}(x, y)(t)-P_{i}(\bar{x}, \bar{y})(t)\right\| \\
& \quad=\left\|g_{i}\left(t, x_{t}, y_{t}\right)-g_{i}\left(t, \bar{x}_{t}, \bar{y}_{t}\right)\right\|
\end{aligned}
$$




$$
\begin{aligned}
& \leq a_{i 1}\left\|x_{t}-\bar{x}_{t}\right\|_{\mathcal{B}_{h}}+a_{i 2}\left\|y_{t}-\bar{y}_{t}\right\|_{\mathcal{B}_{h}} \leq l a_{i 1}\|x-\bar{x}\|_{b}+l a_{i 2}\|y-\bar{y}\|_{b} \\
& \leq l\left(a_{i 1}+a_{i 2}\right)\|(x-\bar{x}, y-\bar{y})\|<\varrho_{i}\|(x-\bar{x}, y-\bar{y})\|<\|(x-\bar{x}, y-\bar{y})\|, \quad i=1,2 .
\end{aligned}
$$

According to (3.14) and (3.15), we conclude that $P$ is contract, namely condition (ii) of Lemma 2.4 holds.

Now we verify that $Q$ is equicontinuous. For all $(x, y) \in \bar{\Omega}, t_{1}, t_{2} \in(-\infty, b]$ with $t_{1}<t_{2}$. When $-\infty<t_{1}<t_{2} \leq 0$, (3.9) and (3.10) give

$$
\left\|\left(Q(x, y)\left(t_{2}\right)-Q(x, y)\left(t_{1}\right)\right)\right\|=\|(0,0)\|=0 \rightarrow 0, \quad \text { as } t_{2} \rightarrow t_{1} .
$$

When $0 \leq t_{1}<t_{2} \leq b$, from (3.9), (3.10), $\left(H_{1}\right),\left(H_{6}\right)$, and $\left(H_{7}\right)$, we have

$$
\begin{aligned}
&\left\|Q_{1}(x, y)\left(t_{2}\right)-Q_{1}(x, y)\left(t_{1}\right)\right\| \\
&=\| \int_{0}^{t_{2}} \mathcal{T}_{\alpha}\left(t_{2}-s\right) f_{1}\left(s, x_{s}, y_{s}, \int_{0}^{s} e_{1}\left(s, \tau, x_{\tau}, y_{\tau}\right) d \tau\right) d s \\
& \quad-\int_{0}^{t_{1}} \mathcal{T}_{\alpha}\left(t_{1}-s\right) f_{1}\left(s, x_{s}, y_{s}, \int_{0}^{s} e_{1}\left(s, \tau, x_{\tau}, y_{\tau}\right) d \tau\right) d s \| \\
&=\| \int_{0}^{t_{1}} \mathcal{T}_{\alpha}\left(t_{2}-s\right) f_{1}\left(s, x_{s}, y_{s}, \int_{0}^{s} e_{1}\left(s, \tau, x_{\tau}, y_{\tau}\right) d \tau\right) d s \\
& \quad+\int_{t_{1}}^{t_{2}} \mathcal{T}_{\alpha}\left(t_{2}-s\right) f_{1}\left(s, x_{s}, y_{s}, \int_{0}^{s} e_{1}\left(s, \tau, x_{\tau}, y_{\tau}\right) d \tau\right) d s \\
& \quad-\int_{0}^{t_{1}} \mathcal{T}_{\alpha}\left(t_{1}-s\right) f_{1}\left(s, x_{s}, y_{s}, \int_{0}^{s} e_{1}\left(s, \tau, x_{\tau}, y_{\tau}\right) d \tau\right) d s \| \\
&=\| \int_{0}^{t_{1}}\left[\mathcal{T}_{\alpha}\left(t_{2}-s\right)-\mathcal{T}_{\alpha}\left(t_{1}-s\right)\right] f_{1}\left(s, x_{s}, y_{s}, \int_{0}^{s} e_{1}\left(s, \tau, x_{\tau}, y_{\tau}\right) d \tau\right) d s \\
& \quad+\int_{t_{1}}^{t_{2}} \mathcal{T}_{\alpha}\left(t_{2}-s\right) f_{1}\left(s, x_{s}, y_{s}, \int_{0}^{s} e_{1}\left(s, \tau, x_{\tau}, y_{\tau}\right) d \tau\right) d s \| \\
& \leq \int_{0}^{b}\left|\mathcal{T}_{\alpha}\left(t_{2}-s\right)-\mathcal{T}_{\alpha}\left(t_{1}-s\right)\right|\left\|f_{1}\left(s, x_{s}, y_{s}, \int_{0}^{s} e_{1}\left(s, \tau, x_{\tau}, y_{\tau}\right) d \tau\right)\right\| d s \\
&+\int_{t_{1}}^{t_{2}}\left|\mathcal{T}_{\alpha}\left(t_{2}-s\right)\right|\left\|f_{1}\left(s, x_{s}, y_{s}, \int_{0}^{s} e_{1}\left(s, \tau, x_{\tau}, y_{\tau}\right) d \tau\right)\right\| d s \\
& \leq {\left[l b\left(B_{11}+B_{12}\right)+l b^{2} B_{13}\left(C_{11}+C_{12}\right)\right]\left|\mathcal{T}_{\alpha}\left(t_{2}-s\right)-\mathcal{T}_{\alpha}\left(t_{1}-s\right)\right| } \\
&+\left[l b N_{1}\left(B_{11}+B_{12}\right)+l b^{2} N_{1} B_{13}\left(C_{11}+C_{12}\right)\right]\left(t_{2}-t_{1}\right) \\
& \rightarrow 0, \quad a s t_{2} \rightarrow t_{1} .
\end{aligned}
$$

Similar to (3.17), we also get

$$
\begin{aligned}
& \left\|Q_{2}(x, y)\left(t_{2}\right)-Q_{2}(x, y)\left(t_{1}\right)\right\| \\
& \leq \quad\left[l b\left(B_{21}+B_{22}\right)+l b^{2} B_{23}\left(C_{21}+C_{22}\right)\right]\left|\mathcal{T}_{\beta}\left(t_{2}-s\right)-\mathcal{T}_{\beta}\left(t_{1}-s\right)\right| \\
& \quad+\left[l b N_{2}\left(B_{21}+B_{22}\right)+l b^{2} N_{2} B_{23}\left(C_{21}+C_{22}\right)\right]\left(t_{2}-t_{1}\right) \\
& \rightarrow 0, \quad \text { as } t_{2} \rightarrow t_{1} .
\end{aligned}
$$


When $-\infty<t_{1}<0<t_{2} \leq b$, noticing that $t_{2} \rightarrow t_{1}$ means that $t_{2} \rightarrow 0^{+}$and $t_{1} \rightarrow 0^{-}$, we obtain

$$
\left\|\left(Q(x, y)\left(t_{2}\right)-Q(x, y)\left(t_{1}\right)\right)\right\| \rightarrow\|(0,0)\|=0, \quad \text { as } t_{2} \rightarrow t_{1} .
$$

From (3.16)-(3.19), we know that $Q$ is equicontinuous. Hence, by the Arzela-Ascoli theorem, we conclude that $Q: \bar{\Omega} \rightarrow \bar{\Omega}$ is completely continuous, that is, condition (iii) of Lemma 2.4 also holds. Thus all the conditions of Lemma 2.4 hold. According to Lemma 2.4 , we know that system (1.1) has a pair of solutions $\left(x^{*}, y^{*}\right) \in \bar{\Omega}$. The proof is completed.

\section{Illustrative example}

Consider the following coupled system of neutral partial integro-differential equations with fractional derivative of the form

$$
\left\{\begin{aligned}
&{ }^{c} D_{t}^{\alpha} {\left[u(t, x)-g_{1}\left(t, u_{t}, v_{t}\right)\right] } \\
&= \frac{\partial^{2}}{\partial x^{2}}\left[u(t, x)-g_{1}\left(t, u_{t}, v_{t}\right)\right] \\
& \quad+f_{1}\left(t, u_{t}, v_{t}, \int_{0}^{t} e_{1}\left(t, s, u_{s}, v_{s}\right) d s\right), \quad x \in[0, \pi], t \in[0,1], \\
& D_{t}^{\beta}\left[v(t, x)-g_{2}\left(t, u_{t}, v_{t}\right)\right] \\
&= \frac{\partial^{2}}{\partial x^{2}}\left[v(t, x)-g_{2}\left(t, u_{t}, v_{t}\right)\right] \\
& \quad+f_{2}\left(t, u_{t}, v_{t}, \int_{0}^{t} e_{2}\left(t, s, u_{s}, v_{s}\right) d s\right), \quad x \in[0, \pi], t \in[0,1], \\
& u(t, x)=\phi_{1}(t, x), v(t, x)=\phi_{2}(t, x), \quad t \in(-\infty, 0], x \in[0, \pi], \\
& u(t, 0)=u(t, \pi)=v(t, 0)=v(t, \pi)=0, \quad t \geq 0,
\end{aligned}\right.
$$

where $0<\alpha, \beta<1, u_{t}=u(t+\theta, x), v_{t}=v(t+\theta, x), \theta \leq 0, \phi_{1}(t, x), \phi_{2}(t, x) \in \mathcal{B}_{h},{ }^{c} D_{t}^{\alpha}$ and ${ }^{c} D_{t}^{\beta}$ are Caputo fractional derivatives.

Let $X=L^{2}[0, \pi]$ with the norm $\|u\|_{L^{2}}=\left(\int_{0}^{\pi}|u(s)|^{2} d s\right)^{\frac{1}{2}}$. $\mathbb{X}=X \times X$ with the norm $\|(u, v)\|=\max \left\{\|u\|_{L^{2}},\|v\|_{L^{2}}\right\}$. We choose $h=e^{2 s}, s<0$, then $l=\int_{-\infty}^{0} h(s) d s=\frac{1}{2}<\infty$ for $t \leq 0$ and determine

$$
\|\phi\|_{\mathfrak{B}_{h}}=\int_{-\infty}^{0} h(s) \sup _{\theta \in[s, 0]}\|\phi(\theta)\|_{L^{2}} d s
$$

Define the operator $\mathscr{A}, \mathscr{B}: D \subset X \rightarrow X$ by $\mathscr{A} w=\mathscr{B} w=w^{\prime \prime}$ with the domain $D$ defined as

$$
D=\left\{w \in X: w, w^{\prime} \text { are absolutely continuous, } w^{\prime \prime} \in X, w(0)=w(\pi)=0\right\} .
$$

Then

$$
\mathscr{A} w=\mathscr{B} w=\sum_{n=1}^{\infty} n^{2}\left\langle w, w_{n}\right\rangle w_{n}, \quad w \in D,
$$

in which $w_{n}(x)=\sqrt{\frac{2}{\pi}} \sin (n x)(n=1,2, \ldots)$ is the orthogonal set of eigenvectors of $\mathscr{A}$ and $\mathscr{B}$. It has been long known that $\mathscr{A}$ and $\mathscr{B}$ are the infinitesimal generators of an analytic 
semigroup $\left\{\mathcal{T}_{\alpha}(t)\right\}_{t \geq 0}$ and $\left\{\mathcal{T}_{\beta}(t)\right\}_{t \geq 0}$ in $X$ and are provided by

$$
\mathcal{T}_{\alpha}(t) w=\mathcal{T}_{\beta}(t) w=\sum_{n=1}^{\infty} e^{-n^{2} t}\left\langle w, w_{n}\right\rangle w_{n}, \quad \forall w \in X, t>0
$$

Since the analytic semigroups $\left\{\mathcal{T}_{\alpha}(t)\right\}_{t \geq 0}$ and $\left\{\mathcal{T}_{\beta}(t)\right\}_{t \geq 0}$ are compact [37], there exist some constants $N_{1}, N_{2}>0$ such that $\left\|\mathcal{T}_{\alpha}(t)\right\|_{\mathcal{L}(X)} \leq N_{1}$ and $\left\|\mathcal{T}_{\beta}(t)\right\|_{\mathcal{L}(X)} \leq N_{2}$.

Take $g_{i}\left(t, u_{t}, v_{t}\right)=\frac{\sin t\left(u_{t}^{2}+v_{t}^{2}\right)}{100}(i=1,2), f_{i}\left(t, u_{t}, v_{t}, w\right)=\frac{u_{t}^{3}+\cos \left(v_{t}\right)+w}{100}(i=1,2), e_{i}\left(t, s, u_{s}, v_{s}\right)=$ $\frac{e^{t+s-2}\left(u_{t}+v_{t}\right)}{100}(i=1,2)$. By the simple calculation, we have

$$
\begin{aligned}
& \left\|g_{i}\left(t, x_{t}, y_{t}\right)-g_{i}\left(t, \bar{x}_{t}, \bar{y}_{t}\right)\right\| \leq \frac{\sqrt{\pi}}{50}\left\|x_{t}-\bar{x}_{t}\right\|_{\mathcal{B}_{h}}+\frac{\sqrt{\pi}}{50}\left\|y_{t}-\bar{y}_{t}\right\|_{\mathcal{B}_{h}}, \\
& \left\|f_{i}\left(t, x_{t}, y_{t}, w\right)-f_{i}\left(t, \bar{x}_{t}, \bar{y}_{t}, \bar{w}\right)\right\| \leq \frac{3 \sqrt{\pi}}{100}\left\|x_{t}-\bar{x}_{t}\right\|_{\mathcal{B}_{h}}+\frac{\sqrt{\pi}}{100}\left\|y_{t}-\bar{y}_{t}\right\|_{\mathcal{B}_{h}}+\frac{\sqrt{\pi}}{100}\|w-\bar{w}\|, \\
& \left\|e_{i}\left(t, s, x_{s}, y_{s}\right)-e_{i}\left(t, s, \bar{x}_{s}, \bar{y}_{s}\right)\right\| \leq \frac{\sqrt{\pi}}{100}\left\|x_{s}-\bar{x}_{s}\right\|_{\mathcal{B}_{h}}+\frac{\sqrt{\pi}}{100}\left\|y_{s}-\bar{y}_{s}\right\|_{\mathcal{B}_{h} .}
\end{aligned}
$$

From the above discussion, we obtain $l=\frac{1}{2}, b=1, a_{11}=a_{21}=a_{12}=a_{22}=\frac{\sqrt{\pi}}{50}, b_{11}=b_{21}=$ $\frac{3 \sqrt{\pi}}{100}, b_{12}=b_{22}=b_{13}=b_{23}=c_{11}=c_{21}=c_{12}=c_{21}=\frac{\sqrt{\pi}}{100}$. Taking $N_{i}=\frac{1}{5}(i=1,2)$, we derive

$$
l\left[a_{i 1}+a_{i 2}+b N_{i}\left(b_{i 1}+b_{i 2}\right)+b^{2} N_{i} b_{i 3}\left(c_{i 1}+c_{i 2}\right)\right] \approx 0.0426<1, \quad i=1,2 .
$$

Thus, conditions $\left(H_{1}\right)-\left(H_{5}\right)$ hold. According to Theorem 3.1, we know that system (4.1) has a pair of unique mild solutions $\left(u^{*}, v^{*}\right) \in L^{2}[0, \pi] \times L^{2}[0, \pi]$. Similarly, we easily verify that conditions $\left(H_{6}\right)-\left(H_{8}\right)$ also hold. It follows from Theorem 3.2 that system (4.1) has at least a pair of mild solutions $\left(u^{*}, v^{*}\right) \in L^{2}[0, \pi] \times L^{2}[0, \pi]$.

\section{Conclusions}

The fractional differential equation has a significant role to play in some phenomena and processes of many fields such as physics, chemistry, aerodynamics, electrodynamics of a complex medium, polymer rheology, capacitor theory, electrical circuits, biology, control theory, fitting of experimental data, and so on. Therefore, the subject of fractional differential equations is gaining much importance and attention. In recent years, some scholars began researching the neutral fractional differential equation. These types of equations usually contain the fractional derivatives of time delay and the abstract operators. So it is difficult and challenging to study them. There are rare papers dealing with a coupled system of neutral fractional equations with time delays. Thereby, we study a class of coupled systems of neutral fractional differential integro-differential equations with infinite delay in a Banach space in this paper. By applying the Banach contraction principle and Krasnoselskii's fixed point theorem, some new existence criteria of mild solutions are obtained.

\section{Acknowledgements}

The authors thank the referees for a number of suggestions which have improved many aspects of this article. 
Availability of data and materials

Not applicable.sd

\section{Competing interests}

The authors declare to have no competing interests.

\section{Authors' contributions}

The authors read and approved the final manuscript.

\section{Author details}

'School of Mathematics and Information Technology, Yuxi Normal University, Yuxi, China. ${ }^{2}$ Department of Applied Mathematics, Kunming University of Science and Technology, Kunming, China.

\section{Publisher's Note}

Springer Nature remains neutral with regard to jurisdictional claims in published maps and institutional affiliations.

Received: 25 March 2019 Accepted: 7 July 2019 Published online: 12 July 2019

\section{References}

1. Baleanu, D., Diethelm, K., Scalas, E., Trujillo, J.: Fractional Calculus: Models and Numerical Methods. World Scientific, Singapore (2012)

2. Dabas, J., Chauhan, A.: Existence and uniqueness of mild solution for an impulsive neutral fractional integro-differential equation with infinite delay. Math. Comput. Model. 57, 754-763 (2013)

3. Santos, J., Arjunan, M., Cuevas, C.: Existence results for fractional neutral integro-differential equations with state-dependent delay. Comput. Math. Appl. 62(3), 1275-1283 (2011)

4. Zhao, K., Wang, K.: Existence of solutions for the delayed nonlinear fractional functional differential equations with three-point integral boundary value conditions. Adv. Differ. Equ. 2016, 284 (2016)

5. Zhao, K.: Triple positive solutions for two classes of delayed nonlinear fractional FDEs with nonlinear integral boundary value conditions. Bound. Value Probl. 2015, 181 (2015)

6. Liao, J., Chen, F., Hu, S.: Existence of solutions for fractional impulsive neutral functional differential equations with infinite delay. Neurocomputing 122, 156-162 (2013)

7. Sakthivel, R., Revathi, P., Ren, Y.: Existence of solutions for nonlinear fractional stochastic differential equations. Nonlinear Anal., Theory Methods Appl. 81, 70-86 (2013)

8. Zhao, K., Gong, P.: Positive solutions of $\mathrm{m}$-point multi-term fractional integral BVP involving time-delay for fractional differential equations. Bound. Value Probl. 2015, 19 (2015)

9. Zhao, K.: Multiple positive solutions of integral BVPs for high-order nonlinear fractional differential equations with impulses and distributed delays. Dyn. Syst. 30(2), 208-223 (2015)

10. Ravichandran, C., Baleanu, D.: Existence results for fractional neutral functional integro-differential evolution equations with infinite delay in Banach spaces. Adv. Differ. Equ. 2013, 215 (2013)

11. Park, J., Balachandran, K., Annapoorani, N.: Existence results for impulsive neutral functional integrodifferential equations with infinite delay. Nonlinear Anal., Theory Methods Appl. 71, 3152-3162 (2009)

12. Guo, T., Jiang, W.: Impulsive fractional functional differential equations. Comput. Math. Appl. 64, 3414-3424 (2012)

13. Zhang, X., Huang, $X .$, Liu, Z.: The existence and uniqueness of mild solutions for impulsive fractional equations with nonlocal conditions and infinite delay. Nonlinear Anal. Hybrid Syst. 4, 775-781 (2010)

14. Cao, J., Luo, Y., Liu, G.: Some results for impulsive fractional differential inclusions with infinite delay and sectorial operators in Banach spaces. Appl. Math. Comput. 273, 237-257 (2016)

15. Bao, H., Cao, J.: Existence of solutions for fractional stochastic impulsive neutral functional differential equations with infinite delay. Adv. Differ. Equ. 2017, 66 (2017)

16. Chang, Y., Anguraj, A., Arjunan, M.: Existence results for impulsive neutral functional differential equations with infinite delay. Nonlinear Anal. Hybrid Syst. 2, 209-218 (2008)

17. Shah, K., Khan, R.: Multiple positive solutions to a coupled systems of nonlinear fractional differential equations. SpringerPlus 2016, 5 (2016)

18. Li, Y., Shah, K., Khan, R.: Iterative technique for coupled integral boundary value problem of non- integer order differential equations. Adv. Differ. Equ. 2017, 251 (2017)

19. Wang, J., Shah, K., Ali, A.: Existence and Hyers-Ulam stability of fractional nonlinear impulsive switched coupled evolution equations. Math. Methods Appl. Sci. 41(6), 2392-2402 (2018)

20. Ali, S., Shah, K., Jarad, F.: On stable iterative solutions for a class of boundary value problem of nonlinear fractional order differential equations. Math. Methods Appl. Sci. 42(3), 968-981 (2018)

21. Shah, K., Khan, R., Baleanu, D.: Study of implicit type coupled system of non-integer order differential equations with anti-periodic boundary conditions. Math. Methods Appl. Sci. (2019). https://doi.org/10.1002/mma.5496

22. Alsaedi, A., Baleanu, D., Etemad, S., Rezapour, S.: On coupled systems of time-fractional differential problems by using a new fractional derivative. J. Funct. Spaces 2016, Article ID 4626940 (2016)

23. Agarwal, R., Baleanu, D., Hedayati, V., Rezapour, S.: Two fractional derivative inclusion problems via integral boundary condition. Appl. Math. Comput. 257, 205-212 (2015)

24. Baleanu, D., Rezapour, S., Mohammadi, H.: Some existence results on nonlinear fractional differential equations. Philos. Trans. R. Soc. Lond. A 371, 20120144 (2013)

25. Baleanu, D., Mohammadi, H., Rezapour, S.: The existence of solutions for a nonlinear mixed problem of singular fractional differential equations. Adv. Differ. Equ. 2013, 359 (2013)

26. Baleanu, D., Mousalou, A., Rezapour, S.: A new method for investigating approximate solutions of some fractional integro-differential equations involving the Caputo-Fabrizio derivative. Adv. Differ. Equ. 2017, 51 (2017) 
27. Baleanu, D., Mousalou, A., Rezapour, S.: On the existence of solutions for some infinite coefficient-symmetric Caputo-Fabrizio fractional integro-differential equations. Bound. Value Probl. 2017, 145 (2017)

28. Baleanu, D., Mousalou, A., Rezapour, S.: The extended fractional Caputo-Fabrizio derivative of order $0 \leq \sigma<1$ on $C_{R}[0,1]$ and the existence of solutions for two higher-order series-type differential equations. Adv. Differ. Equ. 2018, 255 (2018)

29. Kojabad, E., Rezapour, S.: Approximate solutions of a sum-type fractional integro-differential equation by using Chebyshev and Legendre polynomials. Adv. Differ. Equ. 2017, 351 (2017)

30. Aydogan, M., Baleanu, D., Mousalou, A., Rezapour, S.: On approximate solutions for two higher-order Caputo-Fabrizio fractional integro-differential equations. Adv. Differ. Equ. 2017, 221 (2017)

31. Aydogan, M., Baleanu, D., Rezapour, S.: On high order fractional integro-differential equations including the Caputo-Fabrizio derivative. Bound. Value Probl. 2018, 90 (2018)

32. Mallika, D., Baleanu, D., Suganya, S., Arjunan, M.: Existence results for fractional neutral integro-differential systems with nonlocal condition through resolvent operators. An. Științ. Univ. 'Ovidius' Constanța, Ser. Mat. 27(1), 107-124 (2019)

33. Kalamani, P., Baleanu, D., Arjunan, M.: Local existence for an impulsive fractional neutral integro-differential system with Riemann-Liouville fractional derivatives in a Banach space. Adv. Differ. Equ. 2018, 416 (2018)

34. Chen, F., Baleanu, D., Wu, G.: Existence results of fractional differential equations with Riesz-Caputo derivative. Eur. Phys. J. Spec. Top. 226(16-18), 3411-3425 (2017)

35. Shiri, B., Baleanu, D.: System of fractional differential algebraic equations with applications. Chaos Solitons Fractals 120, 203-212 (2019)

36. Chang, Y:: Controllability of impulsive functional differential systems with infinite delay in Banach spaces. Chaos Solitons Fractals 33, 1601-1609 (2007)

37. Kilbas, A., Srivastava, H., Trujillo, J.: Theory and Applications of Fractional Differential Equations. North-Holland Mathematics Studies, vol. 204. Elsevier, Amsterdam (2006)

38. Podlubny, I.: Fractional Differential Equations. Academic Press, New York (1993)

39. Haase, M.: The Functional Calculus for Sectorial Operators. Operator Theory: Advances and Applications, vol. 169. Birkhäuser, Basel (2006)

\section{Submit your manuscript to a SpringerOpen ${ }^{\circ}$ journal and benefit from:}

- Convenient online submission

- Rigorous peer review

- Open access: articles freely available online

- High visibility within the field

- Retaining the copyright to your article

Submit your next manuscript at $\boldsymbol{~ s p r i n g e r o p e n . c o m ~}$ 